\title{
Social inclusion through libraries that provide digital health information and support
}

\author{
Silvia Anton
}

The Museums Libraries and Archives Council (MLA) recently published two research reports that emphasise the role for libraries in supporting social inclusion through greater access and support in obtaining health information and support online.

There are 12.5 million adults in the United Kingdom that do not use the internet and those not using it risk missing out on the full benefits of digitally delivered public services (BIS, 2010). While some choose "digital self-exclusion", others experience "involuntary exclusion", and it has been estimated that these people represent four million people and are the least advantaged members of society (The Independent, 2010).

Many libraries realise that they can make a positive contribution to the national and local health and inequality targets. They can respond to a national government agenda in prioritising health improvement in those who are "socially disadvantaged", as well as local partnership development.

MLA's recent survey of English authorities shows that one hundred and eleven public libraries reported a total of 1,109 current activities related to health and well-being, with a further 107 in preparation at the time of a comprehensive authority-wide survey (Hicks et al., 2010a). Approximately three quarters of all the existing health related activities in libraries are delivered in partnership.

In a separate MLA study (CFE, 2010), libraries have been found to play a strong role in supporting and promoting digital participation

- $\quad 79 \%$ of England's library services offer free internet access all the time;

- $\quad$ There are 33,000 computers across the 152 library authorities and

- $\quad 91 \%$ of library services help people to get online through one-on-one and/or group training sessions.

MLA's comprehensive survey of health activities combined with the evidence of libraries' strong capacity to deliver digital services gives great confidence in the role libraries have in providing digital health information and support. Many core elements of the libraries' health offer such as health information, signposting and health promotion are underpinned by the library digital offer.

Evidence from MLA's recent health study (Hicks et al., 2010a) also indicates that health and social care partners are keen to capitalise more on the potential of libraries to bridge the digital divide. They recognise libraries for providing a number of benefits, in particular that they offer a neutral, nonstigmatised, non-clinical space, and access to individuals that can be 'hard to reach'.

A specific good practice example that highlight the benefits of providing health support in library spaces is illustrated in the partnership between the Manchester library service and the Macmillan Cancer Charity (Hicks et al., 2010b).

The services offered through the library include provision of cancer information and support, and health promotion. The service is based in three community libraries, which provide a range of the Macmillan and Cancer Research UK resources giving quality information about cancer awareness and living with cancer. There are also smaller information points in four community libraries, with plans to extend this. A drop-in support service is offered once a week at each of the three main library 
bases, with additional support available by appointment (face to face or over the phone). Project staff offer information and advice, emotional support, and referrals or signposting to other services such as qualified counselling.

Macmillan feels that there are some clear benefits to delivering cancer services in libraries (Hicks et al., 2010b).The community setting plays a key part in normalising the view of cancer as a health issue. Expertise in community outreach enables messaging to be matched to the locality, supporting targeted health messages appropriate to the local community. A particular focus of Manchester's library service efforts has been on providing digital health information, for example, computerised cognitive behavioural therapy (cCBT) and training library staff to help navigate and increase access to health websites including NHS Choices. NHS Choices has recently won an award for their Libraries Inclusion Programme, which aims to improve the health of groups through digital engagement programmes in libraries.

Computerised CBT is a self-help computer package that looks at ways patients with mild to moderate depression can cope with their condition. One recent programme called Beating the Blues ${ }^{\mathrm{TM}}$ has been endorsed by the National Institute of Health and Clinical Excellence (NICE) and has been shown to be successful for individuals experiencing symptoms of mild to moderate depression. Generally, cCBT service provision in libraries is at an early stage of development, and the MLA survey showed that out of 107 libraries 23 (21\%) run a cCBT scheme, with a further $11(10 \%)$ libraries preparing for CCBT, and that it has potential to be developed further. The endorsement of NICE in treating depression through computer packages combined with MLA's recent evidence of libraries being perceived as safe spaces by both patients and partners is an encouraging sign for the future development of this service.

Anybody uses the library ... you could be there for any reason ... it's a bit anonymous I suppose for people who want to be. (Library user perspective)

A different partnership and delivery model to that adopted in much of Manchester's health partnership work is that of Self Help Services (SHS). SHS provides cCBT services in Manchester and the surrounding area. It is a Manchester based, user-led mental health charity offering self help initiatives and services to people affected by mental health problems. It receives funding from a range of organisations, including the local PCT, to support their work.

SHS feels that libraries provide good venues for the delivery of cCBT therapy because they are free, provide IT resources and support and will also often provide indirect funding through support services such as publicity and printing of cCBT programmes. The level of support offered varies from library to library; some offer support services such as printing free of charge, others charge at a reduced rate. All libraries that SHS has worked with have provided accommodation and use of the computers free. Other community venues may charge for these services. Moreover, SHS values the fact that libraries offer a non-stigmatised, anonymous community space as well as access to computers. The community associations of the library space are important in that it normalises the process for many users, supports social inclusion and breaks down the isolation that many people in need of cCBT therapy are experiencing.

The challenge ahead is to make the offer visible to a broader range of social and health care partners, and by doing that reaching out to a greater number of people who face isolation and potential and/or actual health risks. 


\section{Reference List}

BIS, (2010), National Plan for Digital Participation, DCMS, online at http://webarchive. nationalarchives. gov. uk/+/http://www.culture.gov.uk/images/publications/National-Plan-Digital-Participation-Final.pdf

CFE. (2010). Role of public libraries in supporting and promoting digital participation, Museum Libraries and Archives Council, London. Online at http://research.mla.gov.uk/evidence/documents/public-librariesand-digital-participation-mla.pdf

Hicks D., Creaser C., Greenwood H., Spezi V., White S., Frude N., (2010a). Public library activity in the areas of health and well-being Final report, Appendix C - Survey of public library authorities, Museum Libraries and Archives Council, London. Online at http://research.mla.gov.uk/evidence/ documents/ Appendix\%20C\%20survey\%20of\%20lib\%20auths\%20and\%20mapping.pdf

Hicks D., Creaser C., Greenwood H., Spezi V., White S., Frude N., (2010b). Public library activity in the areas of health and well-being Final report Appendix D - Case studies, Museum Libraries and Archives Council, London.Online at http://research.mla.gov.uk/evidence/ documents/Appendix\%20D\%20case\% 20studies.pdf

The Independent, (2010). Decoding Britain's digital divide, The Independent [newspaper online version], http://www.independent.co.uk/life-style/gadgets-and-tech/ features/decoding-britains-digitaldivide-1887395.html

\section{Biographical Note}

Silvia Anton is the Senior Research Manager at the Museums, Libraries and Archives Council, and was formerly, at the Communities and Local Government Department and the Health Services Research Unit at the University of Aberdeen. Her interests include health systems, community involvement and cultural sector engagement in the health and well-being agenda. 
